\title{
СИСТЕМНЫЙ АНАЛИЗ ВЗАИМОСООТВЕТСТВИЯ СВОЙСТВ ЦИФРОВОГО ПРЕДПРИЯТИЯ И ПРЕДПРИЯТИЙ-КОМПЛИМЕНТОРОВ В РАМКАХ ИНФОРМАЦИОННОЙ СРЕДЫ
}

\author{
(C) 2021 Мугутдинов Рашид Магомедрасулович \\ Высшая инженерно-экономическая школа, соискатель \\ Санкт-Петербургский политехнический университет Петра Великого, Россия, Санкт-Петербург
}

Конкурентоспособность цифрового предприятия базируется на его представленности в информационной среде. Потенциальный потребитель вынужден анализировать актуальный информационный срез, состоящий из информации, описывающей как цифровое предприятия, так и его ТРУ крайне многомерно. Информацию о самом предприятии в рамках данного информационного среза потенциальный потребитель воспринимает в сравнении с информацией, посвящённой основным конкурентам предприятия, посвященной предприятиям-комплементорам, а также общей контекстуальной информации. Особую значимость приобретает восприятие предприятия в сравнении с его комплименторами. В рамках данной статьи представлен комплексный инструментарий системного анализа взаимосоответствия свойств цифрового предприятия и его предприятийкомплименторов в рамках информационной среды.

Ключевые слова: цифровое предприятие, информационная среда, предприятия-комплементоры, системный анализ, конкурентоспособность.

Так как предприятия-комплименторы производит ТРУ дополняющие ценность ТРУ анализируемого предприятия, особую значимость приобретает как сила, так и характер связи данных предприятий в рамках информационной среды. Формируемый в моменте информационный срез, анализируемый потенциальным потребителем, может быть дифференцирован на следующие информационные блоки, в разрезе основных направлений восприятия предприятия относительно его комплименторов:

- Ассоциативный информационный блок. Данный информационный блок отражает совокупность информации, содержательно единообразной (или относительно содержательно единообразной) для предприятия и его ключевых предприятий-комплементоров. Единообразие данной информации трансформируется в сознании потенциального потребителя в своего рода ассоциацию, или ассоциативную связь, следствием чего является условное перенесение сознанием потребителя свойств предприятиякомплементора на исследуемое предприятия.

- Косвенно ассоциативный информационный блок. Данный информационный блок отражает совокупность информации, описывающей содержательно единообразное (или относительно содержательно единообразное) взаимодействие предприятия и его ключевых предприятий- комплементоров с внешней средой. Ключевое отличие данного информационного блока от ассоциативного информационного блока заключается в том, что первый направленного генерируется непосредственно предприятием и его предприятиями-комплементорами, в то время как второй генерируется иными субъектами внешней среды. Основным источником потребления данной информации являются новостные ресурсы.

- Обратно ассоциативный информационный блок. Данный информационный блок отражает совокупность информации, содержательно обратной (или относительно содержательно обратной) для предприятия и его ключевых предприятий комплементоров. Данная информация формирует в сознании потребителя обратную связь между предприятиями-комплементорама и исследуемым предприятием. Во многом данная информация может иметь общеконтекстуальный характер, отражая дифференциацию реакции предприятия на те или иные проявления во внешней среде.

- Косвенно обратно ассоциативный информационный блок. Данный информационный блок отражает совокупность информации, генерируемой иными субъектами внешней среды относительно предприятия и его предприятий комплементоров, содержательно обратной друг 
другу. Как и в случае с косвенно ассоциативным информационным блоком, основными источниками данной информации выступают новостные ресурсы.

- Диссоциативный информационный блок. Данный информационный блок отражает остаточную совокупность информации. Данный блок является наиболее насыщенным, так как, не являясь прямыми конкурентами, исследуемое предприятие и предприятия-комплементоры генерируют самостоятельные информационные потоки, которые могут быть связаны либо описанием единого процесса потребления, либо единым контекстом.

Приведенные блоки информационного среза могут рассматриваться как в моменте, так и в динамике. При этом совокупность первичных относительных квантификаторов позволяет пространственно рассмотреть комплексные свойств как предприятия, так и его конкурентов в сопоставлении с комплементорами. Таким образом в первую очередь необходимо оценить относительное относительные свойства совокупности ассоциативной информации и совокупности обратно ассоциативной информации. Как отмечалось ранее, данная информация генерируется непосредственно предприятием и его комплименторам. При этом данные массивы могут быть тематически дифференцированы. Следовательно процесс сопоставления можно представить иерархически. На первом уровне данные информационные потоки дифференцируются на содержательно-тематические кластеры, состоящие из единообразного массива токенов, дифференцированных в соответствии с удельным весом, отражающим степень принадлежности к соответствующему кластеру. Каждый из выделяемых содержательно тематических кластеров уникален и может быть описан, однако для целей сопоставления это не требуется. На втором уровне производится сравнение каждого из содержательно-тематических кластеров, сформированных на основе информационного потока предприятия, с каждым из содержательно-тематических кластеров, сформированных на основе информационного потока предприятия-комплементора. Данное сравнение может производиться на основании количества единообразных токенов, скорректированного на удельные веса каждого токена. При этом необходимо учитывать, что полное сопоставление токенов нецелесообразно, так как даже по результатам лемматизации, токены обладающие единым содержанием, могут быть морфически дифференцированы. Для целей относительно сравнения токенов может быть использовано расстояние Левенштейна, отражающее минимальное количество операций вставки одного символа, удаления одного символа и замены одного символа на другой, необходимых для превращения одного токена в другой. Таким образом, степень единообразия ассоциативной информации может быть оценена на максимально достигаемая сумма произведений удельного веса токенов с небольшим расстоянием Левенштейна. Математически данная метрика представлена формулой (1).

$$
\begin{aligned}
I_{a s_{u}}=\sum_{1}^{n} \mathrm{q}\left(t_{i}^{x}\right) * \mathrm{q}\left(t_{j}^{y}\right), \\
\text { при } \max \left(d\left(t_{i}^{x} ; t_{j}^{y}\right)\right)
\end{aligned}
$$

Где:

1. $I_{a s_{u}}$ - индекс ассоциативности информационного потока, гарнируемого исследуемым предприятием и информационного потока, гарнируемого предприятием-комплеметором u.

2. $\mathrm{q}\left(t_{i}^{x}\right)$ - удельный вес токена i в содержательно-тематическом кластере х, сформированном на основе информационного потока, гарнируемого исследуемым предприятием.

3. $\mathrm{q}\left(t_{j}^{y}\right)$ - удельный вес токена j в содержательно-тематическом кластере у, сформированном на основе информационного потока, гарнируемого предприятиемкомплеметором $\mathrm{u}$.

4. $d\left(t_{i}^{x} ; t_{j}^{y}\right)$ - расстояние Левенштейна между токеном і в содержательно-тематическом кластере х, сформированном на основе информационного потока, гарнируемого исследуемым предприятием и токеном j в содержательнотематическом кластере у, сформированном на основе информационного потока, гарнируемого предприятием-комплеметором $\mathrm{u}$.

5. n - количество токенов в содержательнотематическом кластере х, сформированном на основе информационного потока, гарнируемого исследуемым предприятием.

Данный параметр рассчитывается для всех возможных сочетаний содержательно тематических кластеров, сформированных на основе информационного потока, гарнируемого исследуемым предприятием и информационного потока, 
гарнируемого предприятием-комплеметором, и в качестве аналитически значимого принимается наибольший. Также данный параметр рассчитывается для комплекса предприятийкомплементоров, что в дальнейшем дает возможность из комплексного сравнения и формулирования решений относительно вектора трансформации информационного потока, генерируемого предприятием.

По результатам расчета комплекса индексов ассоциативности предприятие может идентифицировать наиболее и наименее коррелирующие с ним предприятия-комплементоры. При этом свойство ассоциативности не связано напрямую со свойством обратной ассоциативности. Обратная ассоциативность определяется прямым противоречием информационных потоков с содержательной точки зрения. Таким образом алгоритмически для целей определения данного параметра в первую очередь необходимо реверсировать массив токенов, сформированный для исследуемого предприятия. Реверсировать данный массив возможно посредствам замены существующих частей речи на их антонимы. При этом удельный вес для антонимов сохраняется. Однако, надо отметить, что прямое реверсирование всех элементов массива нецелесообразно, так как это в значительной мере исказит базовую содержательную составляющую (суть основной деятельности), вследствие чего предлагается реверсировать исключительно прилагательные, насыщающие входной массив токенов. Математически данная метрика представлена формулой (2).

$$
\begin{aligned}
I_{\text {re.asu }}=\sum_{1}^{n} \mathrm{q}\left(r e . t_{i}^{x}\right) * \mathrm{q}\left(t_{j}^{y}\right), \\
\text { при } \max \left(d\left(r e . t_{i}^{x} ; t_{j}^{y}\right)\right)
\end{aligned}
$$

Где:

1. $I_{r e . a s_{u}}$ - индекс обратной ассоциативности информационного потока, гарнируемого исследуемым предприятием и информационного потока, гарнируемого предприятием-комплеметором $\mathrm{u}$.

2. $\mathrm{q}\left(r e . t_{i}^{x}\right)$ - удельный вес реверсивного токена і в содержательно-тематическом кластере $\mathrm{x}$, сформированном на основе информационного потока, гарнируемого исследуемым предприятием.

Сопоставление нормированных значений индекса ассоциативности и индекса обратной ассоциативности информационного потока позволяет сформировать двумерный график. Данное графическое сопоставление позволяет проанализировать наиболее содержательно близких для предприятия комплементоров, что в свою очередь дает возможность концентрировать информационный поток, повышая тем самым уровень ассоциативности. При этом стратегия управления информационным потоком также может быть дифференцирована. На рисунке 1 представлено базовое состояние предлагаемого инструмента.

Так как основой матрицы выступают нормированные значения индекса ассоциативности и индекса обратной ассоциативности информационного потока, центром графика является усредненное значение данных показателей по аналитической выборке. Предприятиякомплементоры могут располагаться в одном из четырех секторов. Для каждого из секторов могут быть сформулированы уникальные выводы, на основе которых могут формулироваться управленческие решения. Каждый из секторов можно охарактеризовать следующим образом:

1. Сектор I консолидирует предприятиякомплементоры со сравнительно низким уровнем как ассоциативности, так и обратной ассоциативности информационного потока. Данные значения анализируемых показателей свидетельствует о том, что данные предприятиякомплементоры существуют в принципиально иной содержательно-тематической парадигме, которая не сопоставима с содержательной парадигмой исследуемого предприятия. Ориентация на данные предприятия в рамках формирования образа предприятия в информационной среде целесообразно исключительно в случае высокой значимости данных предприятий в сознании потребителя, однако ориентация на данные предприятия-комплементоры будет требовать значительной трансформации текущего образа предприятия в информационной среде, что вероятнее всего может привести к потери связи с текущими потребителями.

2. Сектор II консолидирует предприятиякомплементоры со сравнительно высоким уровнем ассоциативности и сравнительно низким уровнем обратной ассоциативности информационного потока. Предприятия-комплементоры, составляющие данный сектор являются ключевыми, так как именно их образ в информаци- 


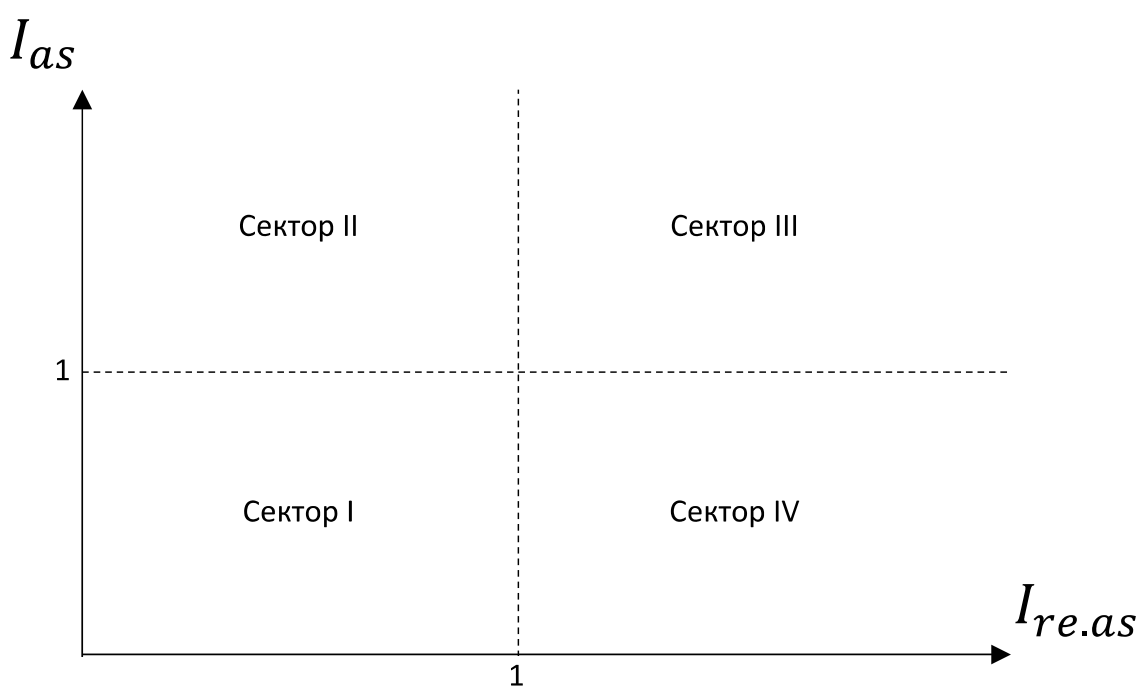

Рисунок 1. Матрица ассоциативности предприятий-комплименторов

онной среде в первую очередь ассоциативен с образом исследуемого предприятия. Следовательно, наиболее эффективным является усиление свойств ассоциативности, что в свою очередь позволит конвертировать успехи и положительные свойства образа данных предприятий в свойства образа исследуемого предприятия в информационной среде. Однако, в случае условно отрицательного информационного потока со стороны данных предприятий-комплементоров, образу исследуемого предприятия в информационной среде также может быть нанесен ущерб. Следовательно, предприятию необходим постоянный мониторинг информационного потока, исходящего со стороны данных предприятийкомплементоров. При этом целесообразным может быть решение о консолидации информационных потоков, что позволит сделать образ данных предприятий-комплементоров в информационной среде более управляемым.

3. Сектор III консолидирует предприятиякомплементоры со сравнительно высоким уровнем как ассоциативности, так и обратной ассоциативности информационного потока. Предприятия-комплементоры, составляющие данный сектор являются наиболее спорными с точки зрения стратегии взаимодействия в информационной среде. Высокий уровень обоих индексов говорит о значительной дифференциации информационного потока, генерируемого данными предприятиями-комплементорами. Одним из наиболее вероятных причин подобно- го является крайне низкий уровень диссоциативного информационного потока, что, в сущности, означает крайне высокий уровень корреляции данного информационного потока с информационным потоком, генерируемым исследуемом предприятием. Следовательно, именно данные предприятия-комплементоры существуют в единой с исследуемым предприятием информационной среде, при этом информационный поток ими генерируемый частично отличается от информационного потока исследуемого предприятия. Таким образом именно данная совокупность предприятий-комплименторов подлежит наиболее детальному анализу и формированию уникальной стратегии взаимодействия, подразумевающей максимизацию полезного эффекта от ассоциативного эффекта и эффекта обратной ассоциации.

4. Сектор IV консолидирует предприятиякомплементоры со сравнительно высоким уровнем обратной ассоциативности, сравнительно низким уровнем ассоциативности информационного потока. Данные предприятиякомплементоры являются «антагонистами» исследуемого предприятия в рамках информационной среды. Следовательно положительный эффект воздействия на потребителей за счет управления информационным потоком данными предприятиями может конвертироваться в отрицательное воздействие на образ исследуемого предприятия. В случае высокой значимости данных предприятий-комплементоров 
в информационной среде необходимо либо трансформировать собственный образ, либо формировать дистанцированый информационный поток, который позволит разрушить связь с данными предприятиями в сознании потребителей.

Анализ соответствующего распределения дает возможность сформулировать как конкретные управленческие решения относительно развития и трансформации образа предприятия в информационной среде, так и сформулировать комплекс рекомендаций и ограничений для формулируемых в дальнейшем управленческих решений. Однако, ассоциации в сознании потребителя формируются не только контролируемым информационным потоком как предприятия, так и его комплементоров, значимая часть образа формируется сторонним информационным потоком. Сторонний информационный поток генерируется в первую очередь источниками новостной информации и аналитической информации. Как отмечалось выше данный поток может быть дифференцирован на косвенно ассоциативный и косвенно обратно ассоциативный. Так как механизм формирования образа в сознании потребителей идентичен в отношении данных информационных потоком, подход к оценке уровня косвенной ассоциативности и косвенной обратной ассоциативности может быть экстраполирован с рассмотренных ранее информационных потоков. Однако, необходимо отметить, что базисом сравнения может выступать информационный поток, генерируемый как предприятием, так и его предприятиямикомплементорами. Следовательно, могут быть выделены следующие сравнительные сочетания:

- Сопоставление направленного информационного потока предприятия и направленного информационного потока его предприятийкомплементов. Данное сравнительное сочетание позволяет оценить непосредственную ассоциативность и обратную ассоциативность, и была рассмотрена ранее.

- Сопоставление стороннего информационного потока, относительно исследуемого предприятия и направленного информационного потока генерируемого непосредственного предприятиями-комплементорами. Данное сопоставление позволит оценить уровень ассоциативности (и обратной ассоциативности) представления предприятия во внешней среде с управляемым образом, генерируемым предприятиями-комплементорами. Данное сопоставление значимо исключительно при высоком уровне представленности того или иного предприятия-комплементора в информационной среде.

- Сопоставление стороннего информационного потока, относительно предприятийкомплементоров и направленного информационного потока генерируемого непосредственного исследуемым предприятием. Данное сопоставление позволит оценить уровень ассоциативности (и обратной ассоциативности) представления предприятий-комплементоров во внешней среде с управляемым образом, генерируемым исследуемым предприятием. Данное сопоставление значимо исключительно при доминирующей роле исследуемого предприятия в информационной среде.

- Сопоставление стороннего информационного потока, относительно предприятийкомплементоров и стороннего информационного потока, относительно исследуемого предприятия. Данное сопоставление позволит оценить уровень ассоциативности (и обратной ассоциативности) представления предприятийкомплементоров во внешней среде с представлением исследуемого предприятия во внешней среде. Данное сопоставление значимо при условно идентичном информационном масштабе исследуемого предприятия и его предприятий-комплементоров, и сущностно является наиболее объективной оценкой косвенной ассоциативности (и обратной косвенной ассоциативности).

Так как данное исследование предполагает сопоставление исключительно информационносоизмеримым предприятий, предлагаемые инструменты оценки базируются на сопоставлении стороннего информационного потока, относительно предприятий-комплементоров и стороннего информационного потока, относительно исследуемого предприятия. Однако надо отметить, что в случае значимой дифференциации масштаба исследуемого предприятия и его предприятий-комплементоров в рамках информационной среды целесообразно произвести соответствующее замещение одного из оцениваемых информационных потоков. Инструменты оценки уровня косвенной ассоциативности и 
косвенной обратной ассоциативности представлены моделями 3 и 4.

$$
\begin{gathered}
I_{a s_{u}}^{i n}=\sum_{1}^{n} \mathrm{q}\left(t_{i}^{x i n}\right) * \mathrm{q}\left(t_{j}^{y \text { in }}\right), \\
\text { при } \max \left(d\left(t_{i}^{x i n} ; t_{j}^{y \text { in }}\right)\right) \\
I_{\text {re.as }}^{\text {in }}=\sum_{1}^{n} \mathrm{q}\left(\text { re.t } t_{i}^{x i n}\right) * \mathrm{q}\left(t_{j}^{\text {yin }}\right), \\
\text { при } \max \left(d\left(\text { re. } t_{i}^{x^{i n}} ; t_{j}^{y \text { in }}\right)\right)
\end{gathered}
$$

Где:

1. $I_{a s_{u}}^{i n}-$ индекс косвенной ассоциативности стороннего информационного потока, относительно исследуемого предприятия и стороннего информационного потока, относительно предприятия-комплементора $\mathrm{u}$.

2. $\mathrm{q}\left(t_{i}^{x i n}\right)$ - удельный вес токена і в содержательно-тематическом кластере х, сформированном на основе стороннего информационного потока, относительно исследуемого предприятия.

3. $\mathrm{q}\left(t_{j}^{\text {yin }}\right)-$ удельный вес токена j в содержательно-тематическом кластере у, сформированном на основе стороннего информационного потока, относительно предприятиякомплементора $\mathrm{u}$.

4. $d\left(t_{i}^{x i n} ; t_{j}^{y \text { in }}\right)$ - расстояние Левенштейна между токеном і в содержательно-тематическом кластере х, сформированном на основе стороннего информационного потока, относительно исследуемого предприятия и токеном $\mathrm{j}$ в содержательно-тематическом кластере у, сформированном на основе стороннего информационного потока, относительно предприятиякомплементора $\mathrm{u}$.

5. n - количество токенов в содержательнотематическом кластере $\mathrm{x}$, сформированном на основе стороннего информационного потока, относительно исследуемого предприятия.

6. Ire.as in $_{u}$ - индекс косвенной обратной ассоциативности стороннего информационного потока, относительно исследуемого предприятия и стороннего информационного потока, относительно предприятия-комплементора $\mathrm{u}$.

7. $\mathrm{q}\left(\right.$ re.t $\left._{i}^{x^{\text {in }}}\right)-$ удельный вес реверсивного токена і в содержательно-тематическом кластеpe $\mathrm{x}$, сформированном на основе стороннего ин- формационного потока, относительно исследуемого предприятия.

Получаемые на основе соотнесения рассчитываемых метрик аналитические результаты могут выступать базисом формирования рекомендаций и ограничений для принятия управленческих решений относительно взаимодействия предприятия с субъектами внешней среды, формирующими данный сторонний информационный поток. Для целей универсализации механизма сопоставления получаемых аналитических метрик, может быть сформирована матрица, конструктивно идентичная матрице ассоциативности предприятий-комплементоров. На рисунке 2 представлен макет матрицы косвенной ассоциативности предприятийкомплименторов.

Данная матрица состоит из четырех секторов, сущностно идентичны секторам матрицы ассоциативности предприятий-комплементоров. Следовательно потенциальные выводы относительно управления представлением предприятия во внешней среде, которые могут быть сформулированы на основе анализа данной матрицы идентичны потенциальным выводам, формулируемым на основе матрицы ассоциативности предприятий-комплементоров. Однако сопоставление распределения предприятий-комплементоров в рамках данных матриц позволит формулировать выводы относительно дифференциации направленного информационного потока, генерируемого предприятиями-комплементорами, и информационного потока, генерируемого иными субъектами информационной среды относительно предприятий-комплементоров. Данная дифференциация также может быть универсализирована с точки зрения рекомендаций и ограничений принятия управлений решений. В частности, могут быть сформулированы следующие сценарии дифференциации:

1. Полное соответствие положения предприятия-комплементора в матрице ассоциативности и матрице косвенной ассоциативности. В данном случае какая-либо дифференциация положения предприятия-комплементора отсутствует, следовательно потенциальные выводы идентичны для обеих матриц.

2. Сектор I матрицы ассоциативности и Сектор ІІматрицы косвенной ассоциативности. В соответствии с данной дифференциацией следует, что предприятие-комплементор формирует 


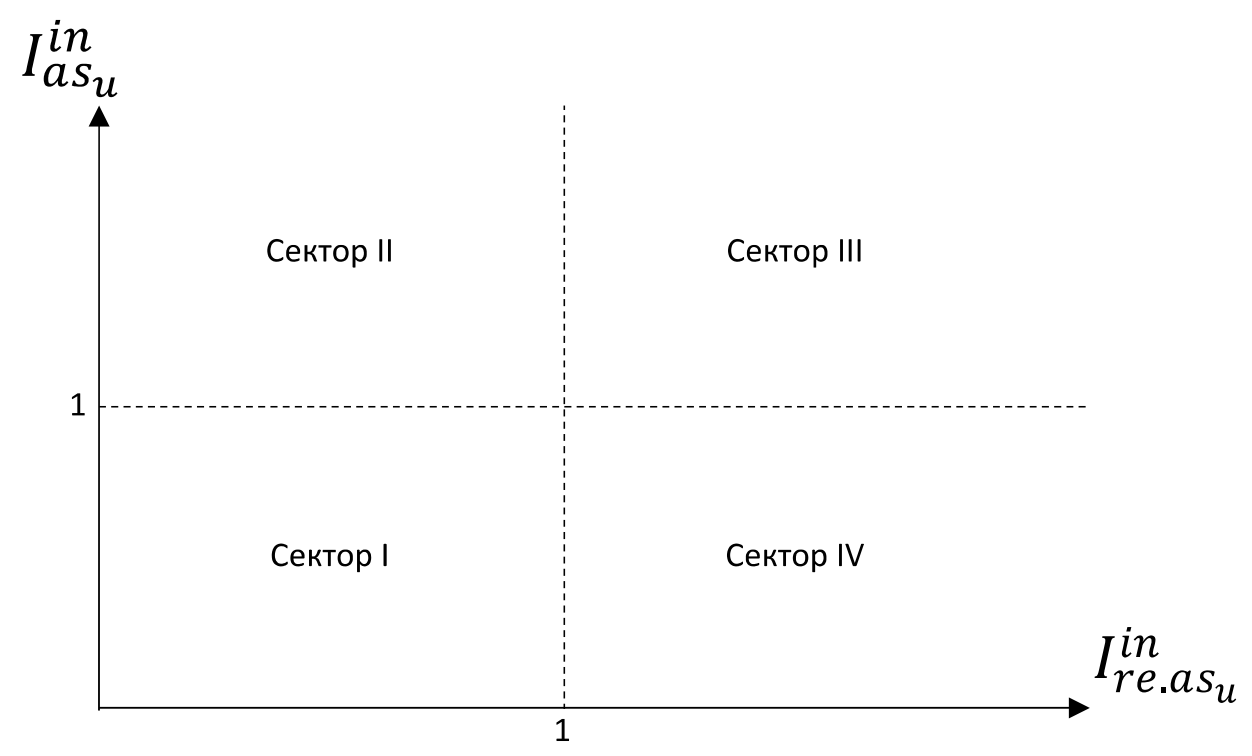

Рисунок 2. Матрица косвенной ассоциативности предприятий-комплименторов

максимально диссоциированный относительно исследуемого предприятия информационный поток, однако сторонний информационный поток, относительно предприятия-комплементора, в достаточной мере ассоциирован со сторонним информационным потоком, относительно исследуемого предприятия. Возможными причинами данной дифференциации могут выступать моментные или системные внешние информационные сопоставления. Данные отклонения могут быть использованы в рамках усиления информационной корреляции между исследуемым предприятием и предприятиями-комплементорами. В частности в рамках совместной маркетинговой деятельности предприятию-комплементору может быть предложена интеграция совместного косвенно ассоциативного информационного блока в направленные информационные потоки. Однако в случае потенциальных маркетинговых потерь от интеграции информационных потоков исследуемому предприятию эффективнее наоборот идентифицировать косвенно ассоциативный информационный блок и воздействовать на исключение его из информационной среды.

3. Сектор I матрицы ассоцииативности $u$ Сектор III матрицы косвенной ассоциативности. В соответствии с данной дифференциацией следует, что предприятие-комплементор формирует максимально диссоциированный относительно исследуемого предприятия информационный поток, однако сторонний инфор- мационный поток, относительно предприятиякомплементора, в достаточной мере как ассоциирован так и обратно ассоциирован со сторонним информационным потоком, относительно исследуемого предприятия. Данная дифференциация является наиболее многомерной с точки зрения потенциальные причин возникновения, а следовательно и с точки зрения направлений воздействия на нее. В данном случае первичным выступает потенциальный эффект от информационной корреляции исследуемого предприятия с предприятием-комплементором. При наличии потенциального положительного маркетингового эффекта от данной корреляции, исследуемому предприятию необходимо формировать единый информационный поток с предприятием-комплементором, в то время как при потенциально отрицательном маркетинговом эффекте предприятию целесообразно как усиливать обратно ассоциативный информационный поток относительно предприятиякомплементора, так и компенсировать ассоциативный информационный поток. Данное информационное многообразие внешней среды может свидетельствовать о низком уровне структурной целостности направленного информационного потока исследуемого предприятия, и следовательно при его структуризации подобный эффект может быть компенсирован.

4. Сектор I матрицы ассоциативности и Сектор IV матрицы косвенной ассоциативности. В соответствии с данной дифференциацией сле- 
дует, что предприятие-комплементор формирует максимально диссоциированный относительно исследуемого предприятия информационный поток, однако сторонний информационный поток, относительно предприятия-комплементора, в достаточной мере обратно ассоциирован со сторонним информационным потоком, относительно исследуемого предприятия. Возможные причины возникновения подобного идентичны причинам дифференциации под номером 2. Однако в данном случае исследуемому предприятию целесообразно компенсировать данный сторонниц информационный поток, в случае значимости предприятия-комплементора в рамках информационной среды. При этом как низкая информационная значимость предприятия-комплементора, так и принципиально отрицательное восприятие предприятиякомплементора основной аудиторией исследуемого предприятия может конвертироваться в положительный маркетинговый эффект. Однако подобное использование отрицательного восприятия потребителей может формировать потенциально неустойчивый спрос, что в последствии может компенсировать моментный маркетинговый эффект.

5. Сектор II матрицы ассоциативности и Сектор І матрицы косвенной ассоциативности. В соответствии с данной дифференциацией следует, что предприятие-комплементор формирует максимально ассоциируемый относительно исследуемого предприятия информационный поток, однако сторонний информационный поток, относительно предприятия-комплементора, диссоциирован со сторонним информационным потоком, относительно исследуемого предприятия. Данная дифференциация является достаточно распространённой в ситуации, когда предприятие-комплементор является партнером исследуемого предприятия и стремится к формированию ассоциативного образа, однако в значительной мере локальная специфика деятельности не позволяет сформировать какой-либо значимый сторонний информационный поток. В данном случае потенциально эффективным с маркетинговой точки зрения является усиление представленности исследуемого предприятия во внешней информационной среде (в стороннем информационном потоке) при активном медиировании сотрудничества с предприятием-комплементором.

6. Сектор II матрицы ассоциативности и
Сектор III матрицы косвенной ассоциативности. В соответствии с данной дифференциацией следует, что предприятие-комплементор формирует максимально ассоциируемый относительно исследуемого предприятия информационный поток, однако сторонний информационный поток, относительно предприятия-комплементора, является в равной мере ассоциируемым и обратно ассоциируемым со сторонним информационным потоком, относительно исследуемого предприятия. Данная дифференциация косвенно свидетельствует о значимости предприятия-комплементора в рамках информационной среды, однако информационный поток предприятия-комплементора либо не в достаточной мере структурирован, либо его объем предполагает значительную дифференциацию информационного позиционирования. В данном случае исследуемому предприятию наиболее целесообразно скорректировать собственный сторонний информационный поток акцентируя ассоциативную часть и компенсируя обратно ассоциативную. В случае обратной корректировки стороннего информационного потока исследуемое предприятие рискует условной информационной концентрацией и формирование определённой «ниши» в рамках информационной среды, что с одной стороны ограничивает потенциальный спрос, а с другой стороны формирует уникальную ценность для потенциального спроса - условную информационную эксклюзивность.

7. Сектор II матрицы ассоциативности и Сектор IV матрицы косвенной ассоциативности. В соответствии с данной дифференциацией следует, что предприятие-комплементор формирует максимально ассоциируемый относительно исследуемого предприятия информационный поток, однако сторонний информационный поток, относительно предприятия-комплементора, является исключительно обратно ассоциируемым со сторонним информационным потоком, относительно исследуемого предприятия. Возможной и наиболее вероятной причиной данной дифференциации может быть принципиальная разница масштабов исследуемого предприятия и предприятия-комплементора в информационной среде. В случае относительного доминирования исследуемого предприятия в информационной среде, какая-либо коррекция управляемого информационного потока не является необходимой, однако, в случае обратного 
для исследуемого предприятия потенциально эффективными являются рекомендации, сформулированные в пункте 6.

8. Сектор III матрицы ассоцииативности и Сектор I матрицы косвенной ассоциативности. В соответствии с данной дифференциацией следует, что предприятие-комплементор формирует единовременно ассоциируемый и обратно ассоциируемый относительно исследуемого предприятия информационный поток, однако сторонний информационный поток, относительно предприятия-комплементора, является диссоциирован со сторонним информационным потоком, относительно исследуемого предприятия. Наиболее вероятной причиной данной дифференциации, также как и в пункте 5, может выступать незначительность масштаба предприятия-комплементора в рамках информационной среды. Следовательно, потенциально наиболее эффективной стратегией исследуемого предприятия является либо игнорирование данной специфики, либо усиление кооперации с предприятием-комплементором и усиление представления данной кооперации в рамках информационной среды.

9. Сектор III матрицы ассоциативности и Сектор II матрицы косвенной ассоциативности. В соответствии с данной дифференциацией следует, что предприятие-комплементор формирует единовременно ассоциируемый и обратно ассоциируемый относительно исследуемого предприятия информационный поток, однако сторонний информационный поток, относительно предприятия-комплементора, является исключительно ассоциированным со сторонним информационным потоком, относительно исследуемого предприятия. Данная дифференциация является одной из наиболее потенциально эффективных с точки зрения кооперации в информационной среде. По сути сторонний информационный поток содержит исключительно ассоциативную часть информационного потока, генерируемого предприятиемкомплементором, и исследуемому предприятию можно рекомендовать непрерывный мониторинг описанной специфики.

10. Сектор III матрицы ассоциативности и Сектор IV матрицы косвенной ассоциативноcmu. В соответствии с данной дифференциацией следует, что предприятие-комплементор формирует единовременно ассоциируемый и обратно ассоциируемый относительно иссле- дуемого предприятия информационный поток, однако сторонний информационный поток, относительно предприятия-комплементора, является исключительно обратно ассоциированным со сторонним информационным потоком, относительно исследуемого предприятия. Данная специфика полностью противоположна специфике, описанной в пункте 9. Следовательно в том случае, если исследуемому предприятию необходима ассоциироваться с предприятиемкомплементором, ему необходимо значимое воздействие на сторонний информационный поток для его кардинальной трансформации. Данная трансформация также будет требовать значимой трансформации управляемого информационного потока, что в свою очередь будет требовать масштабных инвестиций.

11. Сектор IV матрицы ассоциативности и Сектор I матрицы косвенной ассоциативноcmu. В соответствии с данной дифференциацией следует, что предприятие-комплементор формирует максимально обратно ассоциируемый относительно исследуемого предприятия информационный поток, однако сторонний информационный поток, относительно предприятия-комплементора, диссоциирован со сторонним информационным потоком, относительно исследуемого предприятия. Возможной причиной данной специфики может в первую очередь выступать незначительный масштаб предприятия-комплементора в рамках информационной среды. В том случае если для исследуемого предприятия потенциально эффективным является обратно ассоциируемый информационный поток, ему необходимо концентрировать в информационном потоке элементы, формирующие данное свойство. В ином случае потенциально наиболее эффективной стратегией выступает игнорирование данной специфики.

12. Сектор IV матрицы ассоциативности и Сектор II матрицы косвенной ассоциативности. В соответствии с данной дифференциацией следует, что предприятие-комплементор формирует обратно ассоциируемый относительно исследуемого предприятия информационный поток, однако сторонний информационный поток, относительно предприятия-комплементора, является исключительно ассоциированным со сторонним информационным потоком, относительно исследуемого предприятия. Описываемая специфика обратна специфике, описанной 
в пункте 7 , и она является одной из наиболее неоднозначным с управленческой точки зрения. Потенциально значимой является стратегия масштабного воздействия на сторонний информационный поток, однако данная стратегия является крайне затратоемкой. В случае относительно незначительных масштабов предприятия-комплементора в рамках информационной среды потенциально эффективным решением может быть игнорирование данной специфики.

13. Сектор IV матрицы ассоциативности и Сектор III матрицы косвенной ассоциативно$\mathrm{cmu}$. В соответствии с данной дифференциацией следует, что предприятие-комплементор формирует обратно ассоциируемый относительно исследуемого предприятия информационный поток, однако сторонний информационный поток, относительно предприятия-комплементора, является в равной мере ассоциируемым и обратно ассоциируемым со сторонним информационным потоком, относительно исследуемого предприятия. Данная специфика указывает на низкий уровень структурированности информационного потока исследуемого предприятия и/или информационного потока предприятиякомплементора. Потенциально эффективным решением со стороны исследуемого предприятия является усиление управляемого информационного потока и направленное воздействие на сторонний информационный поток с целью его структурирования.

Описанные сценарии дифференциации являются первичными в рамках формирования управленческих решений, направленных на повышение отдачи от управления информационным потоком. Однако описанный инструментарий требует алгоритмизации и автоматизации.

\section{Библиографический список}

1. Мазилкина Е.И., Паничкина Г.Г. Управление конкурентоспособностью Издательство: Омега-Л Год: 2009, С. 27.

2. Конников, Е.А. Маркетинг некропроизводств / Е. А. Конников, О.А. Погребова // Вестник факультета управления СПбГЭУ.-2017. - № 1-1.- С. 277-280.

3. Родионов, Д.Г. Системный анализ конкурентоспособности цифрового предприятия в рамках информационной среды / Д. Г. Родионов, Е. А. Конников, Р. М. Мугутдинов // Экономические науки. - 2020. - № 193. - С. 394-401.- DOI 10.14451/1.193.394.

4. Родионов, Д.Г. Методология системного анализа информационной среды / Д.Г. Родионов, Е.А.Конников, О.А. Конникова // Экономические науки. - 2021. - № 196. - С. 160-174. - DOI 10.14451/1.196.160.

5. Родионов, Д.Г. Информационный капитал предприятия как целевой показатель развития в рамках цифровых экономических систем / Д. Г. Родионов, Е. А. Конников, Д. А. Алферьев // Экономические науки. - 2020.№ 190.- C. 131-137.- DOI 10.14451/1.190.131.

6. Родионов, Д.Г. Влияние информационной среды на субъекты малого и среднего предпринимательства / Д.Г. Родионов, С.В.Ялымов, Е.А.Конников // Экономические науки.-2020.- № 189.- С. 86-91.- DOI $10.14451 / 1.189 .86$.

7. Харламов, А.В. Конкуренция и защита экономических интересов предприятия / А. В.Харламов // СанктПетербургский государственный университет экономики и финансов. Научная сессия профессорскопреподавательского состава, научных сотрудников и аспирантов по итогам НИР 2000 года: Сборник докладов. Общеэкономический факультет, Санкт-Петербург, 01 марта - 302000 года / Редакторы: А. М. Игнатьев, Л. А. Миэринь, А. И.Попов. - Санкт-Петербург: Санкт-Петербургский государственный университет экономики и финансов, 2001.- С. 25-26.

8. Харламов, А.В. Конкурентоспособность инновационной экономики / А.В.Харламов // Социальноинновационная модель хозяйственной системы: проблемы и перспективы построения в России: научная сессия профессорско-преподавательского состава, научных сотрудников и аспирантов по итогам НИР 2007 г.: сборник докладов, Санкт-Петербург, 01 марта - 302008 года / Федеральное агентство по образованию, Санкт-Петербургский государственный университет экономики и финансов; редколлегия: А. Л. Тарасевич, Л. А. Миэринь, А.И. Попов.- Санкт-Петербург: Санкт-Петербургский государственный университет экономики и финансов, 2008. - С. 18-24.

9. Харламов, А.В. Новая конкурентоспособность хозяйствующих субъектов и проблема обеспечения экономической безопасности / А. В.Харламов, К. В. Адаменко // Известия Санкт-Петербургского государственного экономического университета. - 2016. - № 2(98). - С. 13-16. 
10. Харламова, Т.Л. Конкурентоспособность экономики и политика модернизации / Т. Л. Харламова // Модернизация экономических систем: опыт и перспективы: Материалы Международной научно-практической конференции, Махачкала, 21-23 апреля 2011 года / Ответственный редактор: Н. С. Аскеров.- Махачкала: Дагестанский государственный университет, 2011.- С. 330-333.

11. Термин: Лемматизация.- Текст: электронный // Система PromoPult: [сайт].- URL: https://promopult.ru/ library/Лемматизация (дата обращения: 14.07.2021). 